\title{
Preface
}

\section{Practical Issues and Updates in Gynecologic Pathology}

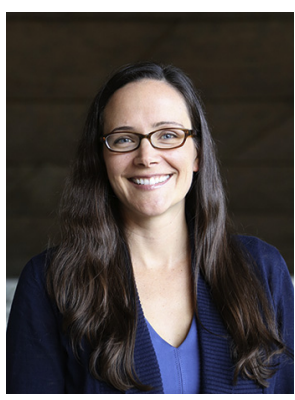

Brooke E. Howitt, MD Editor
I am pleased to serve as the editor for this issue of Surgical Pathology Clinics, focusing on selected practical issues and updates in gynecologic pathology. Gynecologic specimens account for a significant volume of general surgical pathology case load and encompass a wide variety of entities frequently encountered in cervical and endometrial biopsies and resections as well as rarely encountered tumors such as uterine sarcomas and germ cell tumors. It is an exciting time to be a gynecologic pathologist as we continue to expand our understanding of molecular underpinnings of gynecologic tumors and preinvasive lesions. However, it can be difficult to stay up-todate when new tumor subtypes are continually emerging and new classification systems are established. While this issue certainly cannot comprehensively address all updates in gynecologic pathology, we collectively touch on a number of relevant issues for the practicing pathologist.

First, there are some recently proposed changes in classification systems in gynecologic pathology that deserve critical review and attention. For example, histotyping in endocervical adenocarcinoma may undergo significant changes to more accurately reflect tumor biology and specifically the association with human papillomavirus (HPV). In addition, we now appreciate that in HPV-associated endocervical adenocarcinoma, the morphologic pattern of invasion is prognostic. For tubo-ovarian high-grade serous carcinoma (HGSC), the diagnosis may not often be challenging, but there is the age-old question of site of origin (fallopian tube vs ovary). The fallopian tube has, over the last 10 to 15 years, garnered much attention and has indeed shifted from a forgotten organ to one of critical importance with regard to the pathogenesis of (so-called) ovarian HGSC. Because we now tend to examine more, if not all, of the fallopian tube, we are also looking more closely. This has allowed us to detect less obvious preinvasive lesions, including atypias and lesions for which we have no name, or we give many names, but importantly do not precisely know the clinical significance of such lesions. In addition, frequent use of neoadjuvant chemotherapy in HGSC has led to a reproducible and clinically relevant scoring of response to chemotherapy, the "chemotherapy response score." In the field of uterine mesenchymal tumors, there has been, in recent years, description of a number of new entities with specific molecular findings, most notably in highgrade endometrial stromal sarcomas and smooth muscle tumors.

Other topics covered in this issue include frequently encountered epithelial neoplasias, including vulvar and cervical squamous lesions, premalignant lesions of the endometrium, classification of high-grade endometrial carcinomas, and both clear cell and endometrioid ovarian carcinomas. The Lower Anogenital Squamous 
Terminology Standardization Project for HPVassociated lesions has resulted in unifying terminology across all anatomic sites and also addresses the use of p16 immunohistochemistry. One review herein is dedicated entirely to intraoperative evaluation and challenging staging issues in endometrial carcinoma and addresses how we can recognize and measure depth of invasion from sites of involvement by adenomyosis. Based on experience from consultation practices, some areas of gynecologic pathology continue to be troublesome despite few changes in diagnosis and classification; these include lowgrade serous neoplasia, specifically drawing the line between noninvasive implants and lowgrade serous carcinoma and classification of ovarian mucinous tumors, sex-cord stromal tumors, and germ cell tumors (the latter being even more difficult to recognize and diagnose outside the ovary).
I would like to thank all of the authors for their wonderful contributions to this issue of Surgical Pathology Clinics. It has been a pleasure to work with and learn from each and every one of you. Many of the authors in this issue are pathologists whose guidance, mentorship, and friendship have been invaluable to my professional development and career as a gynecologic pathologist, whether across a hallway or across the country (and beyond!).

Brooke E. Howitt, MD

Department of Pathology

Stanford University School of Medicine

300 Pasteur Drive H2128E

Stanford, CA 94305-5324, USA

E-mail address:

bhowitt@stanford.edu 